\title{
Safety Assistance Visual Surveillance Robot
}

\author{
Sachin K, Jasmine Batra, Manisha Mayuree, Gloria Tess Joseph, Sanjay Kumar Singh
}

\begin{abstract}
There is a need for safety assistance visual surveillance that can be effectively used to navigate hazardous places which cannot be accessed by human beings. Several high-risk conditions like radioactive zone, toxic environment and accident-prone areas are usually approached/tackled by humans with little to no information about their conditions. Hence our aim is to reduce any human interaction with these unsafe circumstances by proposing a visual surveillance robot that is capable of moving in any terrain and can relay live information to the controller situated at a remote location. In this paper we address the implementation of Visual Surveillance bot by using a Camera that rotates at 360 degree with the help of DC motor, which illustrate the surrounding so as to provide the estimation of danger if any. We present the execution by efficiently live streaming information with the help of Raspberry pi and by using the MATLAB software to create a RADAR plot by analyzing the object detected by Ultrasonic sensor. The usage of MATLAB not only simplifies the analysis but also helps in creating an enhanced RADAR system by using an ARDUINO to support the ultrasonic system in recording the echo time and object detection angle.
\end{abstract}

Keywords: Android app, Arduino UNO, Bluetooth module, MATLAB, Surveillance.

\section{INTRODUCTION}

Safety in today's world has become the most critical concern for human life. Since in earlier days, humans were involved in safety, and they would easily adapt to any environment they were put into, even risking their life [10]. Citing for such reasons, robots were developed. A large proportion of robotics innovation examine today is given to work the hazardous condition. A robot with a high surveillance system is to be used so that it alarms about the danger giving prior information about the events which cannot be captured through the conventional security systems [4][3]. Therefore, our paper propounds visual security monitoring to improve the old security systems. A robotic system with all the necessary sensors is able to achieve its goal without much time wastage [9]. Considering the flexibility of the robot to adapt to any environment, robots are deployed for

Revised Manuscript Received on July 22, 2019.

* Correspondence Author

Sachin K*, B Tech. in Electronics and Communication Engineering, Vellore Institute of Technology, Vellore, India.

Jasmine Batra, B Tech. in Electronics and Communication Engineering, Vellore Institute of Technology, Vellore, India.

Manisha Mayuree, B Tech. in Electronics and Communication Engineering, Vellore Institute of Technology, Vellore, India.

Gloria Tess Joseph, B Tech. in Electronics and Communication Engineering, Vellore Institute of Technology, Vellore, India.

Prof. Sanjay Kumar Singh, Associate Professor in the Department of Communication Engineering of School of Electronics Engineering at Vellore Institute of Technology, Vellore, India.

(c) The Authors. Published by Blue Eyes Intelligence Engineering and Sciences Publication (BEIESP). This is an open access article under the CC BY-NC-ND license (http://creativecommons.org/licenses/by-nc-nd/4.0/) surveillance to scan the area ceaselessly, providing information if any aberrant event happens [9]

\section{COMPONENTS USED}

\section{A. ARDUINO UNO}

ATmega3298P is the single-chip microcontroller of Arduino R3. It contains an 8-bit RISC processor core. It includes a $32 \mathrm{~kb}$ ISP flash memory with read-while-write capabilities. Arduino works on a voltage between $1.8 \mathrm{~V}-5.5 \mathrm{~V}$ [1]. It is easily available and used in appliances since it is a very low-cost microcontroller. The micro controller is supported by Arduino UNO by providing all the assistance. AC to DC adapter or battery is need for it to start [2]. UNO is different from its previous boards because it doesn't use FTDI USB required for the serial driver. Although Atmega16U2 is programmed as a USB to serial converter is used [2].

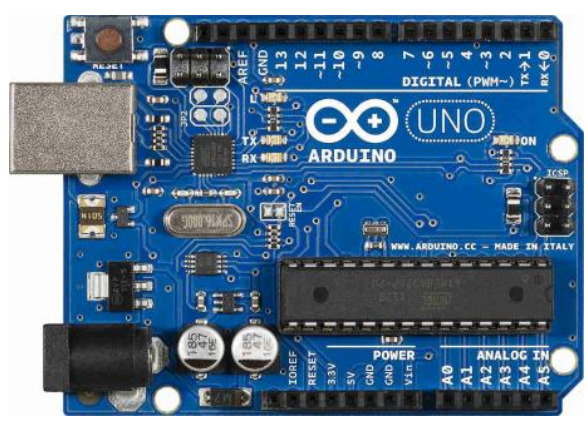

Fig 1: Arduino Uno Board

\section{B. HCO5 BLUETOOTH MODULE}

The Bluetooth module can detect the object up to $100 \mathrm{~m}$. It serially communicates with the microcontroller [1]. HC-05 has slave mode as a default communication, but it can perform tasks on the master, slave, or master/slave mode. The slave mode accepts connection from other modules, but cannot initiate a connection to another Bluetooth device. It is possible for the master connection to initiate a connection between other Bluetooth devices [2]. It has an operating voltage between $4 \mathrm{~V}$ to $6 \mathrm{~V}$; mostly, it is $+5 \mathrm{~V}$, thus enabling the receiver pin of the Bluetooth module to connect with the transmission pin of Arduino. Android application is able to develop a connection if HC-05 is in slave mode. A default password of module " 0000 " or " 1234 " is to be used to maintain the connection between android application and module [1].

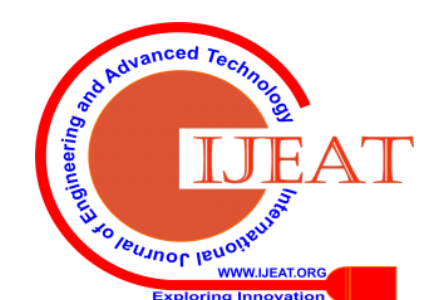




\section{Safety Assistance Visual Surveillance Robot}

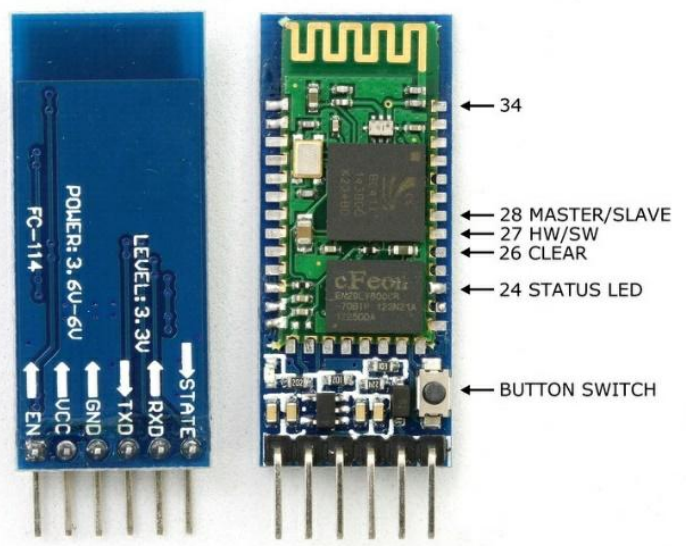

Fig 2: HC05 Bluetooth Module

\section{ULTRASONIC SENSOR}

The ultrasonic sensor measures the distance to an object from 0 to $2.5 \mathrm{~m}$. It operates by generating high-frequency sound and then receiving and checking the properties of the echo pulse. The distance of the object is calculated by measuring the time interval between sending the signal and receiving the signal. Arduino board is connected to the ultrasonic sensor, which is mounted on the servo motor. The rotation of the servo motor provides the sensor with a wider range. Arduino Uno is connected to a laptop which has MATLAB and ARDUINO IDE Software [3]. With the help of these two software, the position of the object is calculated and is displayed.

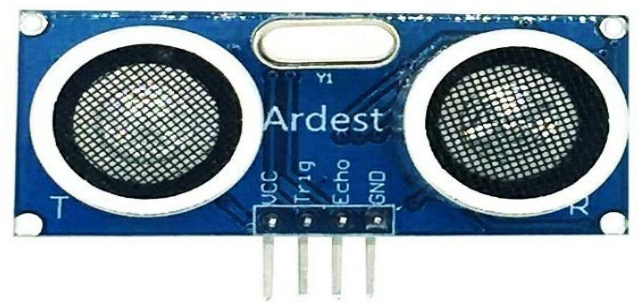

Fig 3: Ultrasonic Sensor

\section{SERVO MOTOR}

It is an electric motor, responsible for mechanical motion that happens around us. A certain amount of electrical signal is given, and the motor rotates at a certain angle, which specify the use of a servo motor. Servo motor will rotate as much as we want and then stop and wait for the next signal for further action [3]. Thus, angular precision is provided by servo motor. Servo motor has vast industrial applications as well as daily life applications such as food blender and electronic devices like DVD tray and machine tools.

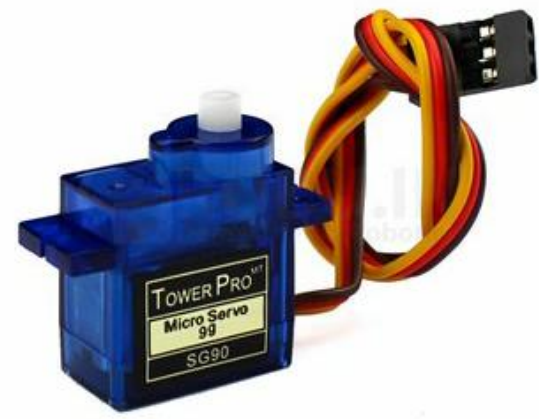

Fig 4: Servo Motor

\section{E. RASPBERRY PI}

It is a credit card sized computer that also functions using a standard keyboard and mouse. The SD card slot acts as the hard drive to Raspberry pi. Raspberry pi operates and controls motion detectors and video cameras for remote sensing and surveillance, streams live video and records it for future playback.[13] The coding is done with the help of Python.

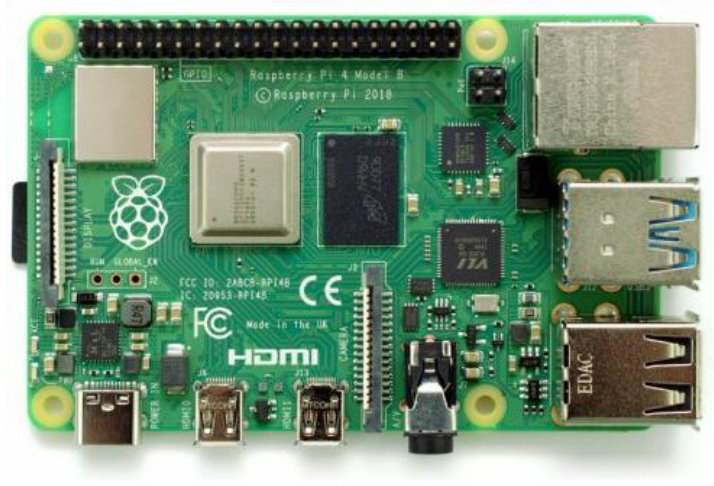

Fig 5: Raspberry Pi

\section{WORKING}

Arduino uno is interfaced with L293D for the forward, backward, left and right movement of the robot and a camera module is used to get live updates to analyses the present situation.[11] The input pins of motor driver are connected to the pins of Arduino. The output generated though this pin will help to rotate the motor in both clockwise and anticlockwise direction. The rotation of motor uses XOR Logic.[15]

Table- I: Direction of Rotation of Motor

\begin{tabular}{|c|c|c|}
\hline Pin $\mathbf{2}$ & Pin 7 & Direction of Rotation \\
\hline 1 & 0 & clockwise \\
\hline 0 & 1 & Anticlockwise \\
\hline 1 & 1 & Stop \\
\hline 0 & 0 & Stop \\
\hline
\end{tabular}

L293D is a 16 pin IC so it can operate two dc motor together. The rotation of left and right motor in clockwise either anticlockwise result in the movement of the robot in all four direction as mentioned in the table.

Table- II: Movement of Robot

\begin{tabular}{|l|l|l|}
\hline Direction & Left Motor & Right Motor \\
\hline Forward & Clockwise & Clockwise \\
\hline Backward & Anti-clockwise & Anti-clockwise \\
\hline Left & Clockwise & Anti-clockwise \\
\hline Right & Anti-clockwise & Clockwise \\
\hline
\end{tabular}

The robot will be given instruction using an android app. These signals will be sent to Arduino using serial communication via Bluetooth module HC05.[15] We are going to control our robot through the $\mathrm{G}$ sensor of our phone using which we can move the robot just by tilting our phone. G-sensor or gravity sensor is commonly referred as accelerometer. It is used in smart phones to control the screen orientation by sensing the change of accelerating force. In this project robot will move in the direction the phone when tilted. We can also use buttons to control the direction of robot .

Published By:

Blue Eyes Intelligence Engineering

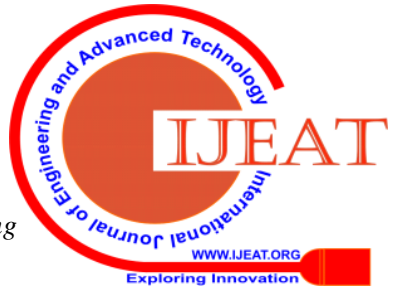


Raspberry pi camera module is used for video surveillance. Camera software in Raspberry pi is enabled and camera is connected to the Pi CSI port. To access video streaming web server IP address of Raspberry pi is required.[12] The video streaming can be monitored on a browser that is connected to the same network as of pi.

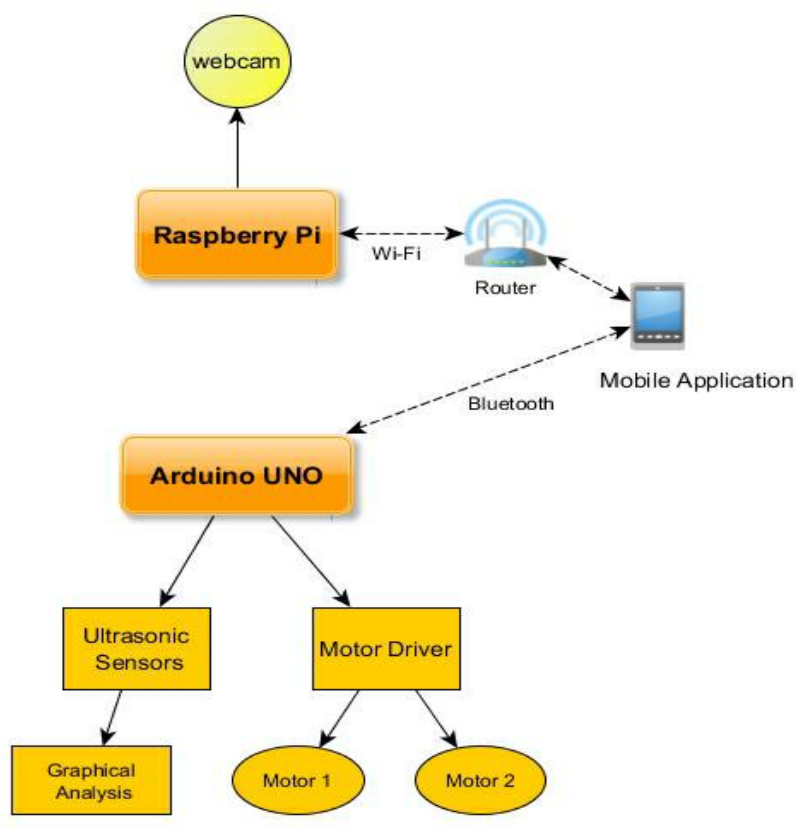

Fig 6: Block Diagram
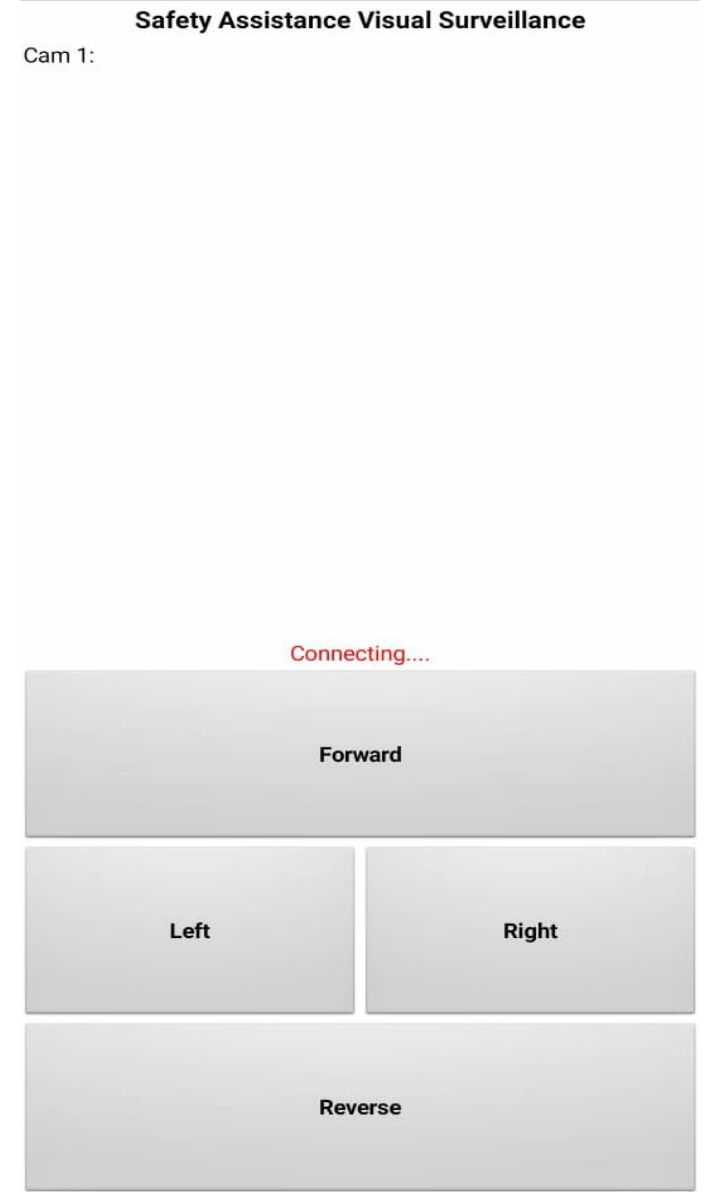

Fig 7: Android Application

\section{RADAR MAPPING USING MATLAB}

Initially the ultrasonic sensor wired to the Arduino board is operated and a pulse of ultrasonic wave is sent. The sensor then responds for any reflected wave that might deflect back from an obstacle [5]. Arduino records this time interval between pulse sent and deflection received and evaluates and preserves this information. It also preserves at what angle the data was observed and then instructs the servo motor to the next position [8]. The board makes the servo motor enhancement of 1 degree from 0 to 180 degree and back to 0 continuously in a loop and simultaneously recording ultrasonic data in centimeters [6][8]. This information is transmitted over to the serial port and is collected by the processor software and after further processing, it plots and updates the RADAR plot [7].

We have successfully enhanced a RADAR system which can detect intrusions and plot the surrounding environment.

The enhanced system detects the obstacle and subsequently plots the changes in position of the obstacle. The ultrasonic sensor mounted on servo motor which provides desired rotation to sensor to increase the range of sensor which is wired to Arduino UNO board [6].Arduino IDE is used to write code and sync to Arduino which helps us to sense position of servo motor and positioning it to the serial port along with the distance of the nearest object in its path [7]. The examine of object detection is completed with the help of MATLAB and therefore using it, we can also trace the position and angle of the object [8].

\section{RESULT ANALYSIS}

The outcome of the ultrasonic radar framework is shown in the figures below. The analysis of object detection is done with the assistance of MATLAB [14]. The plot shows the two-colored regions i.e., 'YELLOW' and 'RED' regions. The yellow part shows that there is no deflection or no object is in the path and red part shows that a deflection has occurred due to an object in a particular region, utilizing this we can likewise trace the position and angle of the object [8].

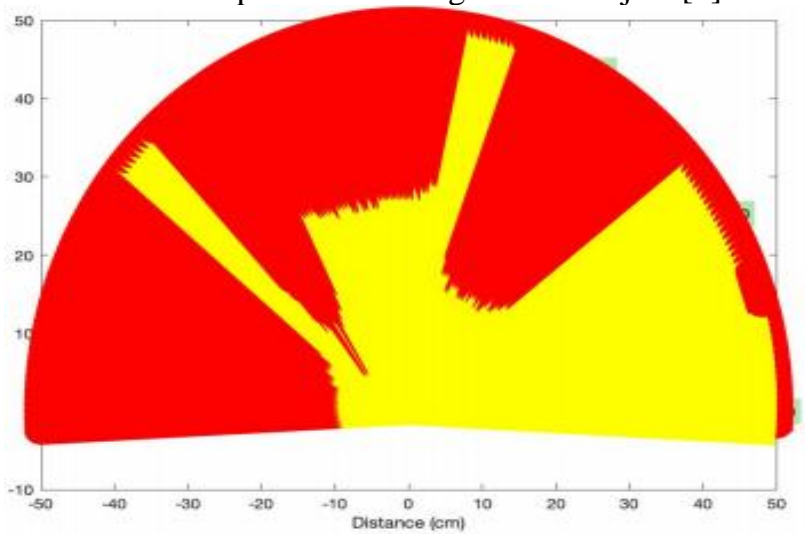

Published By:

Blue Eyes Intelligence Engineering

\& Sciences Publication 

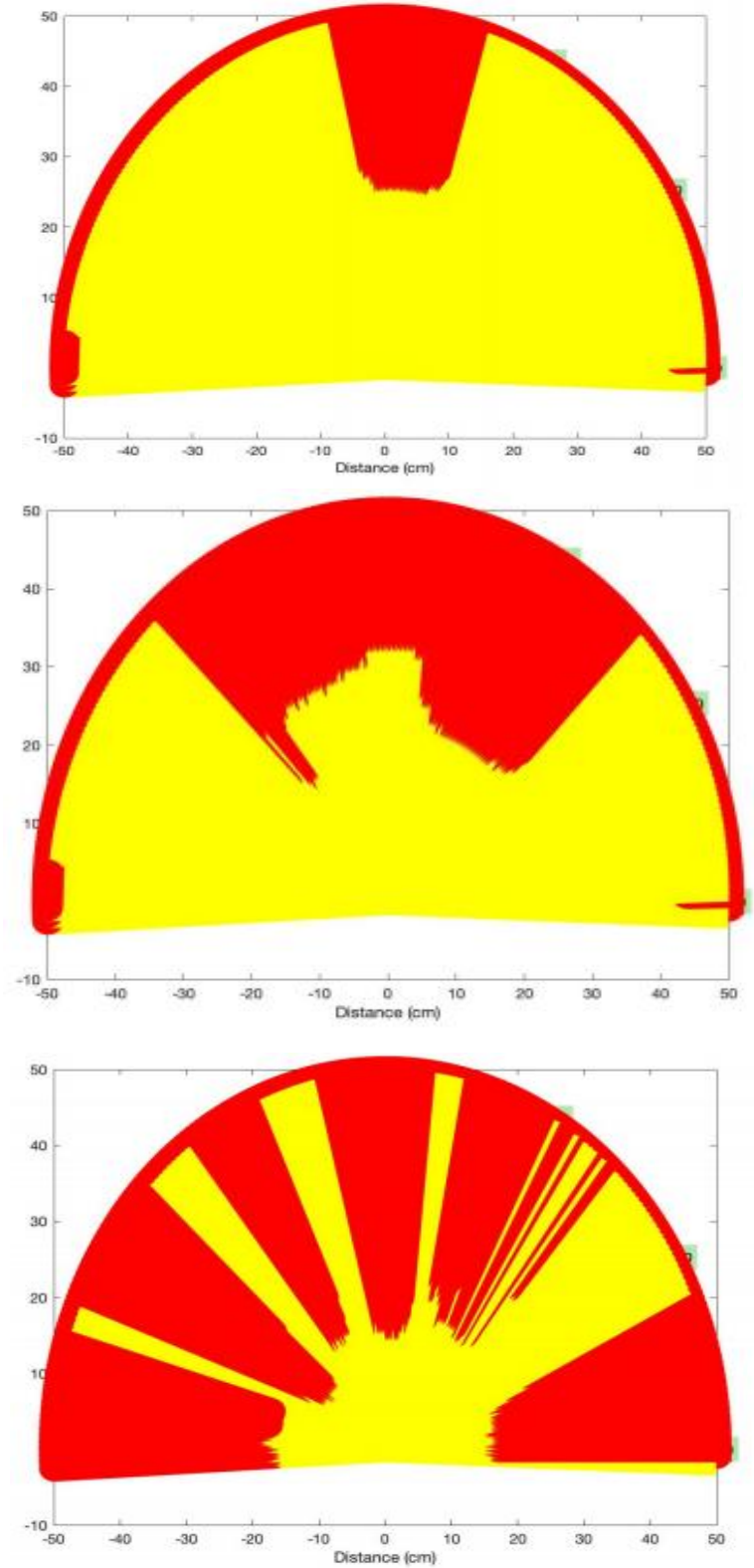

Fig 8: Radar Plots

\section{CONCLUSION AND FUTURE WORK}

This paper presented the importance of visual surveillance of unknown territories and analyzing them before any human intervention. The system is configured using an Arduino, ultrasonic sensor that detects obstacles on its path and can also be used for intrusion detection for location sizes. While servomotor is responsible for the mechanical motion, the RADAR subsystem simultaneously plots the deflection onto the ultrasonic radar framework. The mobile phones now are enhancing into more advanced features, importantly Bluetooth and other applications being a pathway to connect other appliances making the process cheap. And thus, making life easier just by controlling the robot without getting into hazardous places. Further work is related to the consideration of enhancing the surveillance device to not only transmit the live stream but also simultaneously analysis the environment that it is in to detect the toxicity levels.

\section{REFERENCES}

1. Aditya Chaudhry, Manas Batra, Prakhar Gupta, Sahil Lamba, Suyash Gupta, "Arduino based voice controlled robot", International

Published By:

Blue Eyes Intelligence Engineering

\& Sciences Publication

(c) Copyright: All rights reserved.

\section{(ICCCIS), 2019.}

2. Shwetha Patil,Abhigna, Arpitha,,Deepthi,,Priyanka, "Voice Controlled Robot Using Labview" , International Conference on Design Innovations for 3Cs Compute Communicate Control, 2018.

3. Prof. S. A Joshi, Aparna Tondarkar, Krishna Solanke, Rohit Jagtap, "Surveillance Robot for Military Application", International Journal Of Engineering And Computer Science, 23939-23944,Volume 7 Issue 5 May 2018.

4. Hou-Tsan Lee, Wei-Chuan Lin, Ching-Hsiang Huang, Yu-Jhih Huang, "Wireless Indoor Surveillance Robot", SICE Annual Conference, Waseda University, Tokyo, Japan,2011.

5. Tedeschi, A., Calcaterra,S. and Benedetto, F, "Ultrasonic RAdar system (URAS): Arduino and virtual reality for a light-free mapping of indoor environments", IEEE Sensors Journal, 2017,17(14), pp.4595-4604.

6. T. P. Rajan, K. K. Jithin, K. S. Hareesh, C. A. Habeeburahman and.A. Jithin, "Range Detection based on Ultrasonic Principle",International Journal of Advanced Research in Electrical, Electronics and Instrumentation Engineering, vol. 3, no. 2, pp. 7638-7643, 2014.

7. He, H. and Liu, J., "The design of ultrasonic distance measurement system based on S3C2410", 2008 International Conference on Intelligent Computation "Technology and Automation (ICICTA) ,IEEE,(Vol. 2, pp. 44-47), 2008.

8. D. B. Kadam, Y. B. Patil, K. V. Chougale and. S. S. Perdeshi, "Arduino Based Moving Radar System", International Journal of Innovative Studies in Sciences and Engineering Technology (IJISSET), vol. 3, no. 4, pp. 23-27,2017.

9. Gabriele Randelli, Daniele Nardi, Luca Iocchi "'User-friendly security robots" ,Proceedings of the 2011 IEEE International Symposium on Safety,Security and Rescue Robotics Kyoto,Japan,November 1-5,2011.

10. Juan J. Bosch, "Safe and Flexible Human-Robot Cooperation in Industrial Applications",IEEE,Ilmenau,Germany,2010.

11. N.Pugazhenthi, K.VinuLakshmi, V.Preneeth, K.Shrivani, "Design and Fabrication of Robot for Surveillance using Arduino", International Journal of Innovative Technology and Exploring Engineering (IJTTEE) ,2278-3075, 2019.

12. Solomon Azariah R, R.Inbamani, "Android Controlled Surveillance Robot Using Firebase", International Journal of Innovative Research in Science, Engineering and Technology, 2319-8753, 2018.

13. Bokade, A.U. and Ratnaparkhe, V.R., 2016, April. Video surveillance robot control using smartphone and Raspberry pi. In 2016 International Conference on Communication and Signal Processing (ICCSP) (pp. 2094-2097). IEEE.

14. S. Björklund, "The design, development and use of a Matlab toolbox for radar modeling, simulation and signal processing," 2013 14th International Radar Symposium (IRS), Dresden, 2013, pp. 1002-1007.

15. V.Vinay Kumar, A.Apoorva Geetanjali, A.Harshitha, M.Srinath, "Spy Survelliance Robot System Using Arduino",2394-0697,VOLUME-5, ISSUE-4, 2018

\section{AUTHORS PROFILE}

Sachin $\mathbf{K}$ is pursuing Bachelors of Technology in Electronics and Communication Engineering from Vellore Institute of Technology, Vellore, India. His research interests include the fields of cryptography, image processing, deep learning, neural networks, robotics and automation.

Jasmine Batra is pursuing Bachelors of Technology in Electronics and Communication Engineering from Vellore Institute of Technology, Vellore, India. Her research interests include the fields of communications systems, embedded system, robotics and automation, neural networks. 


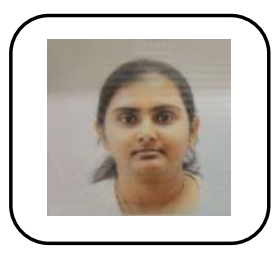

Manisha Mayuree is pursuing Bachelors of Technology in Electronics and Communication Engineering from Vellore Institute of Technology, Vellore, India. Her research interests include the fields of signal processing, image processing, robotics and automation, Internet of Things. She also published a paper on Automatic Plant Watering System in IEEE explore Digital Library

Gloria Tess Joseph is pursuing Bachelors of Technology in Electronics and Communication Engineering from Vellore Institute of Technology, Vellore, India. Her research interests include the fields of communications systems, VLSI design, signal processing, image processing, robotics and automation.

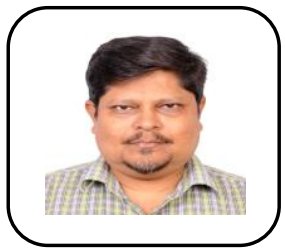

Prof. Sanjay Kumar Singh is an Associate Professor in the Department of Communication Engineering of School of Electronics Engineering at Vellore Institute of Technology, Vellore. He was previously an Associate Professor of Electronics and Communication Engineering at Raghu Engineering College, Visakhapatnam (2015 -2016). He also served as an Assistant Professor (2010-2015) and as a lecturer (2003-2006) in the Department of Electronics and Telecommunication Engineering at College of Engineering Roorkee, Roorkee. He is actively involved in various research activities of Institutions/ Industries, supervised a number of projects at various academic levels and published a large number of research papers in Journals/ Conferences. His main research interests are in the thrust areas of Electronics and Communication Engineering to discover the design principles and instantiate them in both interactive and automated design tools based on concepts like Digital Image Processing, Computer Vision, Machine Learning, Robotics, Internet of Things, Compressed Sensing, Sparse Coding, Independent Component Analysis, Fuzzy Logic, Biological Neural Networks and, X-Lets. 